\title{
PERFIL DOS IDOSOS USUÁRIOS DAS ACADEMIAS AO AR LIVRE PARA A TERCEIRA IDADE
}

\section{Profile of elderly users of outdoor gyms for aged people \\ Perfil de ancianos usuarios de gimnasios al aire libre para la tercera edad}

\author{
Wesley Lessa Pinheiro \\ Faculdade de Medicina da Universidade Federal do Ceará - FAMED/UFC - Fortaleza (CE) - Brasil \\ João Macedo Coelho Filho \\ Faculdade de Medicina da Universidade Federal do Ceará - FAMED/UFC - Fortaleza (CE) - Brasil
}

\section{RESUMO}

Objetivo: Descrever o perfil dos idosos usuários das academias ao ar livre para a terceira idade de Fortaleza, Ceará. Métodos: Estudo transversal, envolvendo uma amostra de 374 idosos com idade maior ou igual a 60 anos, realizado entre os meses de outubro de 2015 e abril de 2016, nas academias ao ar livre para a terceira idade em Fortaleza, Ceará. Aplicou-se um questionário contendo questões sobre dados sociodemográficos, condições de saúde e hábitos de vida autorrelatados, e sobre a utilização dos equipamentos. Mensurou-se o peso e a altura para a obtenção do índice de massa corporal (IMC). Resultados: A maioria dos entrevistados era mulher (56,7\%, $\mathrm{n}=212)$ e com faixa etária entre 60 e 69 anos $(66,8 \%, n=250)$. As principais condições de saúde autorrelatadas foram hipertensão $(40,9 \%$, $n=153)$, colesterol alto $(32,4 \%, n=121)$ e osteoartrite $(26,2 \%, n=98)$. Entre os hábitos de vida, o consumo de bebida alcoólica $(31,6 \%, n=118)$ foi o mais relatado. Verificou-se que $42,8 \%(n=160)$ encontrava-se com excesso de peso. A maioria utilizava os equipamentos há mais de um ano $(68,2 \%, n=255)$, até 30 minutos por dia $(49,5 \%, \mathrm{n}=185)$, cinco ou mais dias durante a semana $(51 \%, \mathrm{n}=191)$, tendo em grande parte percebido melhora na saúde $(93,6 \%, n=350)$. Conclusão: Os idosos apresentaram um perfil jovem e ativo, cujas condições de saúde de maior prevalência podem ser facilmente manejadas com a prática de atividade física, e apresentam características semelhantes independentemente da situação econômica em que se encontram inseridos.

Descritores: Saúde do Idoso; Promoção da Saúde; Serviços de Saúde.

\section{ABSTRACT}

Objective: To describe the profile of elderly users of the outdoor gyms in the city of Fortaleza, Ceará. Methods: Descriptive cross-sectional study, comprising a sample of 374 elderly subjects aged 60 years or above, conducted between October 2015 and April 2016, in the outdoor gyms in the city of Fortaleza, Ceará. A questionnaire was applied with questions addressing sociodemographic data, self-related clinical conditions, lifestyle and the use of workout equipment. Weight and height were measured in order to obtain the body mass index (BMI). Results: A majority of the interviewed subjects were women $(56.7 \%, n=212)$ and 60 to 69 years old $(66.8 \%, n=250)$. The main self-related conditions were hypertension $(40.9 \%, n=153)$, high cholesterol $(32.4 \%, n=121)$ and osteoarthritis $(26.2 \%, n=98)$. Among the life habits, alcohol consumption $(31.6 \%, n=118)$ was the most reported one. It was found that $42.8 \%(n=160)$ were overweight. A majority had been using the workout equipment for at least a year $(68.2 \%, n=255)$, exercising up to 30 minutes a day $(49.5 \%, n=185)$, five or more days a week $(51 \%, n=191)$ and most of them had perceived health improvement $(93.6 \%, n=350)$. Conclusion: The elderly presented a young and active profile, whose most prevalent health conditions can be easily managed with regular physical activity practice, and showed similar characteristics regardless of the economic context in which they are inserted.

Descriptors: Health of the Elderly; Health Promotion; Health Services. 


\section{RESUMEN}

Objetivo: Describir el perfil de ancianos usuarios de gimnasios al aire libre para la tercera edad de Fortaleza (Ceará). Métodos: Estudio transversal con una muestra de 374 ancianos de 60 años o más, entre los meses de octubre de 2105 y abril de 2016, en los gimnasios al aire libre para la tercera edad de Fortaleza, Ceará. Se aplicó un cuestionario con preguntas sobre los datos socio demográficos, las condiciones de salud y los hábitos de vida auto relatados y sobre la utilización de los equipos. Se mensuró el peso y la altura para el cálculo del Índice de Masa Corporal (IMC). Resultados: La mayoría de los entrevistados eran mujeres (56,7\%, $n=212)$ en la franja de edad entre 60 y 69 años $(66,8 \%, n=250)$. Las principales condiciones de salud auto relatadas fueron la hipertensión $(40,9 \%, n=153)$, el colesterol elevado (32,4\%, $n=121)$ y la osteoartritis $(26,2 \%, n=98)$. Entre los hábitos de vida, el consumo de bebida alcohólica $(31,6 \%, n=118)$ ha sido el más relatado. Se verificó que el 42,8\% (n=160) tenía exceso de peso. La mayoría utilizaba los equipos hacía más de un año $(68,2 \%, n=255)$, hasta 30 minutos al día $(49,5 \%, n=185)$, cinco o más días a la semana $(51 \%, n=191)$ y gran parte ha percibido mejora de la salud $(93,6 \%, n=350)$. Conclusión: Los ancianos presentaron un perfil joven y activo cuyas condiciones de salud más prevalentes pueden ser manejadas con la práctica de actividad física y presentan características que no dependen de la situación económica que tienen.

Descriptores: Salud del Anciano; Promoción de la Salud; Servicios de Salud.

\section{INTRODUÇÃO}

Verifica-se, no Brasil, um intenso processo de transição, marcado pelo crescimento da população idosa. A população idosa brasileira, que, no ano 2000, compreendia 14,2 milhões de indivíduos, aumentou para 19,6 milhões em 2010, e projeções mostram que, até o ano de 2030, esse número deve atingir 41,5 milhões $^{(1)}$. O aumento dessa população traz desafios importantes para o Sistema Único de Saúde (SUS), principalmente em decorrência de uma transição epidemiológica prolongada, em que o crescimento dos fatores de risco para as doenças crônicas não transmissíveis encontra grande expressão( ${ }^{(2)}$.

A prática regular de atividade física exerce um papel fundamental no controle e na prevenção das doenças crônicas não transmissíveis, resultando na melhora da mobilidade, da capacidade funcional e da qualidade de vida à medida que se envelhece ${ }^{(3)}$. A adoção de um estilo de vida ativo, torna-se, fundamental para se envelhecer com saúde e com qualidade, principalmente se associado a hábitos alimentares saudáveis. No entanto, mesmo tendo em vista todos os seus benefícios, as pessoas se tornam menos ativas à medida que envelhecem ${ }^{(4)}$.

Estima-se que a inatividade física seja responsável mundialmente por $6 \%$ de doenças cardiovasculares, $7 \%$ de diabetes tipo $2,10 \%$ de cânceres de mama e $10 \%$ de cânceres de cólon, contribuindo para $9 \%$ de mortes prematuras ${ }^{(5)}$. Assim, investir em ações de promoção da atividade física enquanto política pública é fundamental, dado que a inatividade física é identificada como um importante fator de risco para as doenças não transmissíveis. Em Fortaleza, segundo dados do VIGITEL (2010), disponibilizados pelo DATASUS, verificou-se que 40,5\% dos indivíduos com idade de 65 anos ou mais eram fisicamente inativos.

Parques e praças são ambientes ideais para a prática de atividade física, uma vez que são facilmente acessíveis pelos cidadãos. A prática de exercícios nesses espaços pode facilitar o ganho de benefícios à saúde em nível físico e psicológico, estando esses locais, ainda, associados a um bom nível de atividade física ${ }^{(6)}$.

Nesse contexto, têm sido implantadas diversas academias ao ar livre para a terceira idade em praças e parques de Fortaleza, sendo formadas por equipamentos com a finalidade de alongar, fortalecer e desenvolver a musculatura, bem como trabalhar a capacidade aeróbica dos idosos. Contudo, o perfil dos idosos que utilizam os equipamentos das referidas academias ainda é desconhecido. Assim, tendo em vista a importância desse programa como estratégia para a promoção da saúde e enfrentamento das doenças não transmissíveis, investigar o perfil dos idosos usuários das academias caracteriza-se como um importante meio para se conhecer melhor o público atingido pela iniciativa, o que pode contribuir para o desenvolvimento de estratégias dentro do projeto, bem como de outros modelos de políticas públicas de atividade física.

Dessa maneira, este estudo teve como objetivo descrever o perfil dos idosos usuários das academias ao ar livre para a terceira idade de Fortaleza, Ceará.

\section{MÉTODOS}

Trata-se de um estudo quantitativo, do tipo transversal, desenvolvido nas academias ao ar livre para a terceira idade, que estão instaladas e em funcionamento em espaços públicos da cidade de Fortaleza, Ceará.

A cidade possui diversas academias ao ar livre em funcionamento. Neste estudo, foram incluídas, por conveniência operacional e visando contemplar uma razoável representatividade das academias nos diferentes bairros da cidade, as onze primeiras academias instaladas. Essas academias contemplam bairros de situações econômicas distintas, com rendas médias pessoais que variam de 341,36 reais a $3.488,25$ reais. 
A população do estudo foi composta por idosos com idade igual ou superior a 60 anos, independente do sexo, que utilizam os equipamentos das academias, tendo frequentado alguma delas pelo período de, no mínimo, um mês. Foram excluídos do estudo indivíduos sem plena condição de raciocínio ou comunicação ou quando em companhia de cuidador ou familiar que informasse a existência de diagnóstico médico para essas condições.

Foram entrevistados 374 indivíduos, cujo tamanho da amostra recebeu cálculo por meio do StatCalc, componente do software estatístico Epi Info 7.1.5.0. A população é correspondente ao número total de idosos que habitam os bairros onde se encontram as academias escolhidas para a realização do estudo, de acordo com dados provenientes do Censo 2010, obtidos por meio de base de dados secundária. Esperou-se um intervalo de confiança de $95 \%$ para uma estimativa de prevalência de $50 \%$ de idosos usuários, uma vez que não foram encontradas informações sobre essa prevalência nesse grupo específico. Dividiu-se a amostra em dois grupos de acordo com a renda média dos bairros onde se encontravam localizadas as academias, sendo "G1" o grupo que utilizava os equipamentos instalados nos bairros de maior renda e "G2" os bairros de menor renda.

Os dados do estudo foram coletados no período entre outubro de 2015 e abril de 2016, de segunda à sexta-feira, no horário de $5 \mathrm{~h}$ da madrugada às $10 \mathrm{~h}$ da manhã e também de $4 \mathrm{~h}$ da tarde às $9 \mathrm{~h}$ da noite, períodos identificados como os com maior frequência de idosos que compõem a população-alvo do estudo. Estes receberam convite pelo pesquisador de maneira casual, de acordo com a facilidade de acesso aos mesmos.

Aplicou-se um questionário padronizado composto por três distintas seções. A primeira seção apresentava questões com a finalidade de identificar dados sociodemográficos (sexo, idade, etnia, estado civil, escolaridade, exercício de atividade profissional e aposentadoria), a segunda, com questões sobre a condição de saúde autorrelatada (hipertensão, diabetes mellitus, colesterol alto, asma, cardiopatia, osteoartrite, osteoporose, ocorrência de queda, tabagismo e consumo de bebida alcoólica), e a terceira seção investigou o padrão de utilização dos equipamentos (tempo de uso, tempo de utilização diária, frequência semanal, frequência aos finais de semana, satisfação com a quantidade dos equipamentos, satisfação com a qualidade dos equipamentos, percepção de segurança, prática adicional de exercício, companhia durante o exercício, apoio da família e percepção de melhora de saúde).

Utilizou-se uma balança digital da marca Oxer e um estadiômetro portátil da marca Sanny para a mensuração da massa corporal e da altura dos entrevistados, utilizados porteriormente para o cálculo do índice de massa corporal (IMC) por meio da divisão da massa do indivíduo pelo quadrado da sua altura. O critério utilizado para a classificação do IMC foi o proposto por Lipschitz ${ }^{(7)}$, que leva em consideração as mudanças na composição corporal que ocorrem em decorrência do processo de envelhecimento.

Os dados coletados foram processados no software estatístico SPSS versão 23.0. Fez-se uso de estatística descritiva bivariada, compreendendo a distribuição das frequências das diferentes categorias discriminadas de acordo com a renda média do bairro. Utilizou-se o teste Qui-Quadrado $\left(\chi^{2}\right)$ de Pearson, com nível de significância de $5 \%(p<0,05)$, para verificar potenciais diferenças estatisticamente significativas entre os grupos. Tendo em vista a diminuição da ocorrência de erros, executou-se um controle de qualidade que pressupôs o mecanismo de revisão de todos os questionários antes da digitação e ainda a digitação dos dados em dupla entrada no software estatístico.

O estudo foi submetido ao Comitê de Ética em Pesquisa da Universidade Federal do Ceará, tendo sido aprovado sob o parecer $n^{\circ} 1.320 .650$. Foram respeitados os preceitos éticos de acordo com a Resolução 466/12, do Conselho Nacional de Saúde, que regulamenta os aspectos éticos e legais da pesquisa em seres humanos.

\section{RESULTADOS}

Foram entrevistados 374 idosos, com média de idade de 67,59 ( \pm 6,29) anos, sendo 89 anos a idade do indivíduo mais velho entrevistado. No que diz respeito ao perfil sociodemográfico da amostra, verifica-se que a maioria era do sexo feminino $(56,7 \%$, $\mathrm{n}=212)$, na faixa etária entre 60 a 69 anos $(66,8 \%, \mathrm{n}=250)$, de cor branca $(66 \%, \mathrm{n}=247)$ e casada $(61 \%, \mathrm{n}=228)$, conforme disposto na Tabela I.

Ao observar o nível de escolaridade dos indivíduos, verifica-se que mais de um terço dos participantes possuía segundo grau completo $(38,5 \%, n=144)$, bem como ensino superior completo $(34,7 \%, n=130)$. Foi encontrada uma proporção de $31,6 \%$ $(\mathrm{n}=118)$ de participantes que ainda exercem alguma atividade profissional. Grande parte era de aposentados ou pensionistas $(77,3 \%, n=289)$. 
Tabela I - Descrição do perfil sociodemográfico dos idosos usuários das academias ao ar livre para a terceira idade discriminado por renda média dos bairros. Fortaleza, Ceará, 2015-2016.

\begin{tabular}{|c|c|c|c|c|c|c|c|}
\hline \multirow{2}{*}{ Variável } & \multicolumn{2}{|c|}{ G1 } & \multicolumn{2}{|c|}{ G2 } & \multicolumn{2}{|c|}{ Total } & \multirow{2}{*}{ p-valor } \\
\hline & n (188) & $\%$ & n (186) & $\%$ & $\mathrm{n}$ & $\%$ & \\
\hline Sexo & & & & & & & 0,906 \\
\hline Masculino & 82 & 43,6 & 80 & 43 & 162 & 43,3 & \\
\hline Feminino & 106 & 56,4 & 106 & 57 & 212 & 56,7 & \\
\hline Faixa etária & & & & & & & 0,290 \\
\hline 60 a 69 & 122 & 64,9 & 128 & 68,8 & 250 & 66,8 & \\
\hline 70 a 79 & 52 & 27,7 & 51 & 27,4 & 103 & 27,6 & \\
\hline 80 ou mais & 14 & 7,4 & 7 & 3,8 & 21 & 5,6 & \\
\hline Etnia & & & & & & & $0,001 *$ \\
\hline Branca & 134 & 71,3 & 113 & 60,8 & 247 & 66 & \\
\hline Afro-descendente & 8 & 4,3 & 29 & 15,6 & 37 & 9,9 & \\
\hline Outras & 46 & 24,4 & 44 & 23,6 & 90 & 24,1 & \\
\hline Estado civil & & & & & & & 0,701 \\
\hline Casado & 112 & 59,6 & 116 & 62,4 & 228 & 61 & \\
\hline Solteiro & 32 & 17 & 27 & 14,5 & 59 & 15,8 & \\
\hline Divorciado & 16 & 8,5 & 20 & 10,7 & 36 & 9,6 & \\
\hline Viúvo & 28 & 14,9 & 23 & 12,4 & 51 & 13,6 & \\
\hline Escolaridade & & & & & & & $0,000^{*}$ \\
\hline Primário incompleto & 8 & 4,3 & 8 & 4,3 & 16 & 4,3 & \\
\hline Primário completo & 10 & 5,3 & 34 & 18,3 & 44 & 11,8 & \\
\hline Colegial incompleto & 18 & 9,6 & 22 & 11,8 & 40 & 10,7 & \\
\hline Colegial completo & 70 & 37,2 & 74 & 39,8 & 144 & 38,5 & \\
\hline Superior completo & 82 & 43,6 & 48 & 25,8 & 130 & 34,7 & \\
\hline Exercício de atividade profissional & & & & & & & 0,879 \\
\hline Sim & 60 & 31,9 & 58 & 31,2 & 118 & 31,6 & \\
\hline Não & 128 & 68,1 & 128 & 68,8 & 256 & 68,4 & \\
\hline Aposentadoria & & & & & & & 0,097 \\
\hline Sim & 152 & 80,9 & 137 & 73,7 & 289 & 77,3 & \\
\hline Não & 36 & 19,1 & 49 & 26,3 & 85 & 22,7 & \\
\hline
\end{tabular}

As condições de saúde autorrelatadas mais frequentes foram hipertensão arterial $(40,9 \%, \mathrm{n}=153)$, colesterol elevado $(32,4 \%, n=121)$, consumo de algum tipo de bebida alcoólica $(31,6 \%, n=118)$ e osteoartrite $(26,2 \%, n=98)$ (Tabela II). 
Tabela II - Descrição das condições de saúde autorrelatadas dos idosos usuários das academias ao ar livre para a terceira idade discriminado por renda média dos bairros. Fortaleza, Ceará, 2015-2016.

\begin{tabular}{|c|c|c|c|c|c|c|c|}
\hline \multirow{2}{*}{ Variável } & \multirow{2}{*}{$\begin{array}{l}\text { G1 } \\
\text { n (188) }\end{array}$} & \multicolumn{3}{|c|}{ G2 } & \multicolumn{2}{|c|}{ Total } & \multirow{2}{*}{ p-valor } \\
\hline & & $\%$ & n (186) & $\%$ & $\mathrm{n}$ & $\%$ & \\
\hline Hipertensão & & & & & & & 0,411 \\
\hline Sim & 73 & 38,8 & 80 & 43 & 153 & 40,9 & \\
\hline Não & 115 & 61,2 & 106 & 57 & 221 & 59,1 & \\
\hline Diabetes mellitus & & & & & & & 0,497 \\
\hline Sim & 24 & 12,8 & 19 & 10,2 & 43 & 11,5 & \\
\hline Não & 164 & 87,2 & 167 & 89,8 & 331 & 88,5 & \\
\hline Colesterol alto & & & & & & & $0,001 *$ \\
\hline Sim & 46 & 24,5 & 75 & 40,3 & 121 & 32,4 & \\
\hline Não & 142 & 75,5 & 111 & 59,7 & 253 & 67,6 & \\
\hline Asma & & & & & & & 0,349 \\
\hline Sim & 4 & 2,1 & 7 & 3,8 & 11 & 2,9 & \\
\hline Não & 184 & 97,9 & 179 & 96,2 & 363 & 97,1 & \\
\hline Cardiopatia & & & & & & & 0,345 \\
\hline Sim & 14 & 7,4 & 19 & 10,2 & 33 & 8,8 & \\
\hline Não & 174 & 92,6 & 167 & 89,8 & 341 & 91,2 & \\
\hline Osteoartrite & & & & & & & 0,443 \\
\hline Sim & 46 & 24,5 & 52 & 28 & 98 & 26,2 & \\
\hline Não & 142 & 75,5 & 134 & 72 & 276 & 73,8 & \\
\hline Osteoporose & & & & & & & 0,571 \\
\hline Sim & 27 & 14,4 & 23 & 12,4 & 50 & 13,4 & \\
\hline Não & 161 & 85,6 & 163 & 87,6 & 324 & 86,6 & \\
\hline Ocorrência de queda (último ano) & & & & & & & 0,850 \\
\hline Sim & 27 & 14,4 & 28 & 15,1 & 55 & 14,7 & \\
\hline Não & 161 & 85,6 & 158 & 84,9 & 319 & 85,3 & \\
\hline Tabagismo & & & & & & & 0,349 \\
\hline Sim & 4 & 2,1 & 7 & 3,8 & 11 & 2,9 & \\
\hline Não & 184 & 97,9 & 179 & 96,2 & 363 & 97,1 & \\
\hline Consumo de bebida alcoólica & & & & & & & 0,550 \\
\hline Sim & 62 & 33 & 56 & 30,1 & 118 & 31,6 & \\
\hline Não & 126 & 67 & 130 & 69,9 & 256 & 68,4 & \\
\hline
\end{tabular}

G1: Maior renda média; G2: Menor renda média; *diferença estatística para $p<0,05$

Verificou-se, ao categorizar o IMC, que 7,5\% $(\mathrm{n}=28)$ dos participantes encontravam-se abaixo do peso, 49,7\% ( $\mathrm{n}=186)$ deles encontravam-se eutróficos, e 42,8\% ( $\mathrm{n}=160$ ) encontravam-se com excesso de peso (Tabela III).

Tabela III - Descrição do perfil antropométrico dos usuários das academias ao ar livre para a terceira idade discriminado por renda média dos bairros. Fortaleza, Ceará, 2015-2016.

\begin{tabular}{|c|c|c|c|c|c|c|c|c|c|c|}
\hline \multirow{2}{*}{ Variável } & \multicolumn{2}{|c|}{ G1 } & \multicolumn{4}{|c|}{ G2 } & \multicolumn{2}{|r|}{ Total } & \multirow[b]{2}{*}{ Média } & \multirow[b]{2}{*}{ p-valor } \\
\hline & n (188) & $\%$ & Média & n (186) & $\%$ & Média & $\mathbf{N}$ & $\%$ & & \\
\hline Peso (kg) & & & 68,95 & & & 69,78 & & & 69,36 & \\
\hline Altura (m) & & & 1,60 & & & 1,60 & & & 1,60 & \\
\hline IMC & & & 26,56 & & & 26,91 & & & 26,73 & \\
\hline Abaixo do peso & 11 & 5,9 & & 17 & 9,1 & & 28 & 7,5 & & \\
\hline Eutrófico & 101 & 53,7 & & 85 & 45,7 & & 186 & 49,7 & & 0,217 \\
\hline Excesso de peso & 76 & 40,4 & & 84 & 45,2 & & 160 & 42,8 & & \\
\hline
\end{tabular}

G1: Maior renda média; G2: Menor renda média.

Em relação ao perfil de utilização das academias, verificou-se que $68,2 \%(n=255)$ utilizava as academias há mais de um ano e que $51 \%(\mathrm{n}=191)$ frequentava a academia ao menos 5 dias na semana. A proporção de usuários satisfeitos com a quantidade dos equipamentos presentes nas academias foi de $73 \%(\mathrm{n}=273)$, e satisfação com a qualidade dos equipamentos compreenderam $62,8 \%(\mathrm{n}=235)$ dos entrevistados. A proporção de idosos que relataram melhora de saúde em decorrência da utilização das academias foi de 93,6\%, (n=350) (Tabela IV). 
Encontraram-se diferenças estatisticamente significativas entre os grupos nas variáveis etnia $(p=0,001)$, escolaridade $(\mathrm{p}=0,000)$ e colesterol alto $(\mathrm{p}=0,001)$.

Tabela IV - Descrição do perfil de padrão de utilização e percepções dos usuários das academias ao ar livre para a terceira idade discriminados por renda média dos bairros. Fortaleza, Ceará, 2015-2016.

\begin{tabular}{|c|c|c|c|c|c|c|c|}
\hline \multirow{2}{*}{ Variável } & \multicolumn{2}{|c|}{ G1 } & \multicolumn{2}{|c|}{ G2 } & \multicolumn{2}{|c|}{ Total } & \multirow{2}{*}{ p-valor } \\
\hline & n (188) & $\%$ & n (186) & $\%$ & $\mathrm{n}$ & $\%$ & \\
\hline Tempo de uso & & & & & & & 0,214 \\
\hline Um a quatro meses & 38 & 20,2 & 36 & 19,3 & 74 & 19,8 & \\
\hline Cinco a oito meses & 13 & 6,9 & 24 & 12,9 & 37 & 9,9 & \\
\hline Nove meses a um ano & 3 & 1,6 & 5 & 2,7 & 8 & 2,1 & \\
\hline Mais de um ano & 134 & 71,3 & 121 & 65,1 & 255 & 68,2 & \\
\hline Tempo de utilização diária & & & & & & & 0,208 \\
\hline Até 30 minutos & 91 & 48,4 & 94 & 50,5 & 185 & 49,5 & \\
\hline De 30 minutos a $1 \mathrm{~h}$ & 78 & 41,5 & 64 & 34,4 & 142 & 38 & \\
\hline Mais de $1 \mathrm{~h}$ & 19 & 10,1 & 28 & 15,1 & 47 & 12,5 & \\
\hline Frequência semanal & & & & & & & 0,331 \\
\hline Menor que 3 dias & 21 & 11,2 & 17 & 9,1 & 38 & 10,2 & \\
\hline 3 ou 4 dias & 66 & 35,1 & 79 & 42,5 & 145 & 38,8 & \\
\hline 5 dias ou mais & 101 & 53,7 & 90 & 48,4 & 191 & 51 & \\
\hline Utilização durante os finais de semana & & & & & & & 0,086 \\
\hline $\operatorname{Sim}$ & 66 & 35,1 & 50 & 26,9 & 116 & 31 & \\
\hline Não & 122 & 64,9 & 136 & 73,1 & 258 & 69 & \\
\hline Satisfação com a quantidade dos equipamentos & & & & & & & 0,452 \\
\hline $\operatorname{Sim}$ & 134 & 71,3 & 139 & 74,7 & 273 & 73 & \\
\hline Não & 54 & 28,7 & 47 & 25,3 & 101 & 27 & \\
\hline Satisfação com a qualidade dos equipamentos & & & & & & & 0,407 \\
\hline Sim & 122 & 64,9 & 113 & 60,8 & 235 & 62,8 & \\
\hline Não & 66 & 35,1 & 73 & 39,2 & 139 & 37,2 & \\
\hline Percepção de segurança & & & & & & & 0,313 \\
\hline Sim & 136 & 72,3 & 143 & 76,9 & 279 & 74,6 & \\
\hline Não & 52 & 27,7 & 43 & 23,1 & 95 & 25,4 & \\
\hline Prática de caminhada & & & & & & & 0,128 \\
\hline Sim & 126 & 67 & 138 & 74,2 & 264 & 70,6 & \\
\hline Não & 62 & 33 & 48 & 25,8 & 110 & 29,4 & \\
\hline Companhia durante a realização dos exercícios & & & & & & & 0,887 \\
\hline $\operatorname{Sim}$ & 122 & 64,9 & 122 & 65,6 & 244 & 65,2 & \\
\hline Não & 66 & 35,1 & 64 & 34,4 & 130 & 34,8 & \\
\hline Incentivo familiar & & & & & & & 0,490 \\
\hline Sim & 121 & 64,4 & 126 & 67,7 & 247 & 66 & \\
\hline Não & 67 & 35,6 & 60 & 32,3 & 127 & 34 & \\
\hline Percebeu melhora de saúde & & & & & & & 0,978 \\
\hline Sim & 176 & 93,6 & 174 & 93,5 & 350 & 93,6 & \\
\hline Não & 12 & 6,4 & 12 & 6,5 & 24 & 6,4 & \\
\hline
\end{tabular}

G1: Maior renda média; G2: Menor renda média.

\section{DISCUSSÃO}

No presente estudo, a maioria dos entrevistados foi de mulheres, indo ao encontro do que foi observado em duas investigações, que encontraram proporções de $75 \%$ e $53,8 \%$ de mulheres que utilizavam os equipamentos de academias ao ar livre respectivamente ${ }^{(8,9)}$. De uma maneira geral, observou-se que os participantes que utilizam os equipamentos das academias são em sua maioria idosos jovens, encontrando-se na faixa etária entre 60 e 69 anos, o que pode ser justificado por um declínio do nível de atividade física com o avançar da idade ${ }^{(10)}$.

A baixa frequência de indivíduos negros no grupo de maior renda média pode ter sido fator determinante para a diferença estatística encontrada entre os grupos. É importante mencionar que minorias étnicas possuem prevalência de inatividade física superior à encontrada em indivíduos brancos ${ }^{(11)}$. A proporção de idosos casados encontrada vai de encontro com a encontrada em outra investigação ${ }^{(9)}$, o que pode ser justificado por uma maior atividade no lazer entre indivíduos que possuem cônjuge em comparação com aqueles que não possuem ${ }^{(12)}$.

Um maior nível educacional dobra a chance de participação de idosos em exercícios ${ }^{(13)}$. Verificou-se que a maior proporção dos entrevistados, em ambos os grupos, possuía alto grau de escolaridade. A diferença encontrada entre os grupos nessa 
variável pode ser explicada pela maior proporção de aposentados com ensino superior no grupo de maior renda média, reflexo da associação entre condição econômica e nível de escolaridade. Os entrevistados, em sua maioria, não exerciam nenhuma atividade profissional, sendo em grande parte aposentados. Tendo em vista o processo de transição para a aposentadoria como determinante para a prática de atividade física ${ }^{(14)}$, faz-se importante aproveitar essa janela de oportunidade no contexto das academias para a manutenção/adesão a um estilo de vida ativo.

Considerando-se o perfil sociodemográfico observado, é necessário o desenvolvimento de estratégias que possibilitem a adesão e a manutenção da prática de atividade física nas academias por parte de participantes em idade mais avançada, nãobrancos, não-casados e com nível mais baixo de escolaridade, em virtude da baixa presença desses indivíduos, bem como intervenções que possibilitem uma maior prática por indivíduos que se encontram exercendo alguma atividade profissional e a adoção de um estilo de vida ativo por parte de idosos aposentados ou pensionistas.

A hipertensão arterial foi a condição clínica relatada mais prevalente na amostra estudada. Um estudo identificou uma prevalência de $40,2 \%$ de hipertensão em idosos fisicamente ativos ${ }^{(15)}$, proporção semelhante à observada nesta investigação. A segunda condição clínica relatada mais prevalente na amostra foi o colesterol alto, com proporção próxima à encontrada em outra investigação, que identificou $32,7 \%$ de hipercolesterolemia entre idosos praticantes de atividade física ${ }^{(16)}$. Foi encontrada, ainda, uma diferença estatisticamente significativa entre os grupos, sendo essa uma condição mais prevalente no grupo de menor renda média.

O consumo de bebidas alcoólicas foi relatado por quase três terços da amostra. Proporção superior à encontrada em dois estudos, que observaram prevalências de $19,9 \%$ e $14,4 \%$ respectivamente ${ }^{(17,18)}$. Dado seu impacto psicossocial e por ser considerado fator de risco para doenças cardiovasculares, diabetes, doenças hepáticas, entre outras ${ }^{(19)}$, verifica-se a necessidade de intervenção de educação em saúde com o público que utiliza os equipamentos das academias. A osteoartrite, outra condição clínica importante, foi relatada por pouco mais de um quarto dos entrevistados. Indivíduos com artrite possuem risco de mortalidade relacionado a eventos cardiovasculares aumentado ${ }^{(20)}$. Assim, deve-se monitorar essa condição nos usuários das academias, buscando potencializar os efeitos cardiovasculares do exercício sobre essa condição.

Ainda que observadas em menores proporções, as demais condições clínicas não devem ser negligenciadas. A ocorrência de asma na população idosa, por exemplo, tornou-se uma preocupação emergente, tendo em vista a carga epidemiológica substancial associada à essa condição ${ }^{(21)}$. Doenças cardiovasculares, por sua vez, são as principais causas de morte em indivíduos de ambos os sexos ${ }^{(22)}$. A ocorrência de quedas encontra-se entre as principais causas de morbidade e mortalidade na população idosa $^{(23)}$. Todas essas condições podem ser tratadas/prevenidas por meio da prática de exercícios.

Identificou-se, no presente estudo, que mais de um terço dos idosos entrevistados apresentavam excesso de peso. Esse dado merece atenção uma vez que o excesso de peso se caracteriza como uma condição que acarreta problemas de saúde em todas as faixas etárias, aumentando o risco de problemas crônico-degenerativos.

Verifica-se assim a necessidade de monitorar o perfil clínico dos idosos periodicamente, com a finalidade de proporcionar a melhora dessas condições e, consequentemente, da qualidade de vida dos usuários dos equipamentos por meio da prática adequada de atividade física.

A capital cearense conta, atualmente, com diversas academias ao ar livre instaladas e em funcionamento em espaços públicos de diversos bairros, sendo algumas implantadas pela gestão pública e outras em parceria com a iniciativa privada. Objetiva-se, por meio da instalação desses espaços, estimular a prática de exercícios físicos ao ar livre, bem como o desenvolvimento de hábitos saudáveis.

No que diz respeito à utilização dos equipamentos das referidas academias, verificou-se que a maioria dos entrevistados, neste estudo, fazia uso dos mesmos há mais de um ano. Em outra investigação, observou-se que 47,6\% dos indivíduos relataram estar utilizando os equipamentos durante esse tempo ${ }^{(9)}$.

Nesta pesquisa quase metade dos participantes utilizava os equipamentos por até 30 minutos, e mais da metade frequentava a academia em média 5 dias ou mais durante a semana. O American College of Sports Medicine (ACSM) indica que sejam praticados 30 minutos de atividade física moderada, 5 dias por semana, totalizando 150 minutos semanais, sendo essa recomendação proposta também por outras organizações como o American Heart Association (AHA) e a Organização Mundial da Saúde (OMS) ${ }^{(24,25)}$. Verifica-se, portanto, que os usuários têm atingido o proposto pelas principais recomendações de atividade física no que diz respeito à frequência semanal, mas não atingindo a recomendação mínima diária. Menos de um terço dos idosos fazia uso dos equipamentos durante o final de semana, proporção menor do que a encontrada em outra pesquisa ${ }^{(9)}$.

Quando questionados sobre a quantidade e a qualidade dos equipamentos disponibilizados, a maioria dos entrevistados relatou estar satisfeita. Deve-se, entretanto, ressaltar a preocupação com os indivíduos que relataram não estarem satisfeitos, dado como o estado de manutenção de algumas estruturas percebidos pelos usuários repercute nos níveis de atividade física ${ }^{(26)}$.

Fortaleza é uma cidade com alta taxa de criminalidade. No ano de 2015, a taxa de crimes violentos letais intencionais na capital cearense foi de 63,9 por 100 mil habitantes ${ }^{(27)}$. Apesar disso, a maioria dos idosos relatou sentir-se segura nos ambientes onde se encontram instaladas as academias, indicando satisfação com questões relacionadas à segurança pública nesses espaços.

A prática de caminhada era comum entre a maioria dos idosos entrevistados. Uma investigação identificou a caminhada como a modalidade mais praticada por idosos no lazer, sendo exercida por $87,7 \%$ dos homens e $63 \%$ das mulheres ${ }^{(28)}$. Outra pesquisa identificou uma frequência de $20,2 \%$ de caminhada no lazer em idosos paulistas ${ }^{(29)}$. 
Neste estudo, pouco mais de um terço dos idosos praticava seus exercícios em companhia de alguém. Em outra investigação, verificou-se que $37,5 \%$ dos frequentadores de academias ao ar livre utilizavam os equipamentos acompanhados ${ }^{(9)}$. A prática de exercícios em companhia de alguém, bem como a presença de outros idosos nos espaços onde se encontram instaladas as academias, pode contribuir para a redução de sintomas depressivos. A depressão é um transtorno relacionado a diversos fatores, como a tristeza, a solidão e o isolamento social. Nesse contexto, a prática de exercícios pode ser bastante significativa para a melhoria do bem-estar, do contato social e da qualidade de vida dos idosos ${ }^{(30)}$. Faz-se importante também mencionar a importância do suporte familiar como motivação para a prática de exercícios ${ }^{(31)}$. Quase dois terços dos idosos entrevistados recebiam incentivo familiar, proporção um pouco maior que a encontrada em outra pesquisa, onde $58,6 \%$ dos frequentadores recebia algum nível de apoio social familiar ${ }^{(9)}$.

A melhora da saúde em decorrência da utilização dos equipamentos foi relatada pela maioria dos idosos. Essa melhora também foi percebida pela maioria dos participantes em outras duas investigações ${ }^{(9,32)}$. É possível, dessa maneira, observar a influência da prática de exercícios na percepção de saúde dos usuários dos equipamentos das academias, contribuindo significantemente para o aumento da qualidade de vida e da saúde desses indivíduos.

Identifica-se, tendo em vista o perfil de padrão de utilização dos usuários, a importância da manutenção apropriada dos equipamentos, de maneira a garantir uma continuidade em relação à prática de exercícios pelos usuários, bem como incentivar a adesão por parte de outros idosos, garantindo segurança e orientação para a execução das atividades.

Destaca-se como limitação deste estudo a incipiência de outras investigações com a finalidade de descrever o perfil de idosos que utilizam equipamentos das academias ao ar livre para efeito de comparação, o que levou, principalmente no que diz respeito às condições clínicas, à utilização de trabalhos desenvolvidos com a população geral. Além disso, por se tratarem de informações autorrelatadas, estão passivas de vieses de aferição, uma vez que não foram realizadas as devidas avaliações.

\section{CONCLUSÃO}

Os idosos usuários das academias ao ar livre apresentam um perfil jovem e ativo, e cujas condições de saúde mais prevalentes podem ser facilmente manejadas por meio da prática regular de atividade física. As poucas diferenças estatísticas encontradas entre os grupos, que utilizam as academias instaladas em localizações de condições econômicas distintas, indicam que têm atingindo a população alvo de maneira uniforme independentemente da situação econômica em que se encontra inserida.

\section{REFERÊNCIAS}

1. Borges G, Campos MD, Silva LC. Transição da estrutura etária no Brasil: oportunidades e desafios para a sociedade nas próximas décadas. In: Ervatti L, Borges GM, Jardim AP. Mudança demográfica no Brasil no século XXI: subsídios para as projeções da população. Rio de Janeiro: IBGE; 2015. p. 138-151.

2. Duarte EC, Barreto SM. Transição demográfica e epidemiológica: a epidemiologia e serviços de saúde revisita e atualiza o tema. Epidemiol Serv Saúde. 2012;21(4):529-32.

3. Matsudo SMM. Envelhecimento, atividade física e saúde. BIS Bol Inst Saúde (Impr). 2009;(47):76-9.

4. Possamai L, Zogo A, Boni J, Jacques M, Dorst L, Dorst D. Fitness for elders: a comparison between practioners and nonpractioners of exercise. Age (Dordr). 2015;37(3):1-8.

5. Lee I-M, Shiroma EJ, Lobelo F, Puska P, Blair SN, Katzmarzyk PT, et al. Effect of physical inactivity on major noncommunicable diseases worldwide: an analysis of burden of disease and life expectancy. Lancet. 2012;380(9838):219-29.

6. Bedimo-Rung AL, Mowen AJ, Cohen DA. The significance of parks to physical activity and public health: a conceptual model. Am J Prev Med. 2005;28(Suppl 2):159-68.

7. Lipschitz DA. Screening for nutritional status in the elderly. Prim Care. 1994;21(1):55-67.

8. Paulo TR. Programa Academia da Terceira Idade no município de Uberaba/MG. Arq Ciências Esporte. 2013;1(1):54-9.

9. Souza C, Fermino R, Añez C, Reis R. Perfil dos frequentadores e padrão de uso das academias ao ar livre em bairros de baixa e alta renda de Curitiba-PR. Rev Bras Ativ Fís Saúde. 2014;19(1):86.

10. Chodzko-Zajko WJ. Exercise and physical activity for older adults. Kinesiology Review. 2014;3(1):101-6.

11. Crespo CJ, Smit E, Andersen RE, Carter-Pokras O, Ainsworth BE. Race/ethnicity, social class and their relation to physical inactivity during leisure time: results from the Third National Health and Nutrition Examination Survey, 1988-1994. Am J Prev Med. 2000;18(1):46-53.

12. Zaitune MPA, Barros MBA, César CLG, Carandina L, Goldbaum M, Alves MCGP. Fatores associados à prática de atividade física global e de lazer em idosos: Inquérito de Saúde no Estado de São Paulo (ISA-SP), Brasil. Cad Saúde Pública. 2010;26(8):1606-18. 
13. Haley C, Andel R. Correlates of physical activity participation in community-dwelling older adults. J Aging Phys Act. 2010;18(4):375-89.

14. Barnett I, van Sluijs EM, Ogilvie D. Physical activity and transitioning to retirement: a systematic review. Am J Prev Med. 2012;43(3):329-36.

15. Modeneze DM, Maciel ES, Vilela GB Júnior, Sonati JG, Vilarta R. Perfil epidemiológico e socioeconômico de idosos ativos: qualidade de vida associada com renda, escolaridade e morbidades. Estud Interdiscip Envelhec. 2013;18(2):387-99.

16. Virtuoso JF, Mazo GZ, Menezes EC, Cardoso ASA, Dias RG, Balbé GP. Perfil de morbidade referida e padrão de acesso a serviços de saúde por idosos praticantes de atividade física Morbidity profile and the standard of access to health services for elderly practitioners of physical activities. Ciênc Saúde Coletiva. 2012;17(1):23-31.

17. Moura EC, Malta DC. Consumo de bebidas alcoólicas na população adulta brasileira: características sociodemográficas e tendência. Rev Bras Epidemiol. 2011;14(Supl 1):61-70.

18. Luz EP, Dallepiane LB, Kirchner RM, Silva LAA, Silva FPd, Kohler J, et al. Perfil sociodemográfico e de hábitos de vida da população idosa de um município da região norte do Rio Grande do Sul, Brasil. Rev Bras Geriatr Gerontol. 2014;17(2):303-14.

19. Geib LTC. Determinantes sociais da saúde do idoso. Ciênc Saúde Coletiva. 2012;17(1):123-33.

20. Tierney M, Fraser A, Kennedy N. Physical activity in rheumatoid arthritis: a systematic review. J Phys Act Health. 2012;9(7):1036-48.

21. Song W-J, Cho S-H. Challenges in the management of asthma in the elderly. Allergy Asthma Immunol Res. 2015;7(5):4319.

22. Mansur ADP, Favarato D. Mortalidade por doenças cardiovasculares no Brasil e na região metropolitana de São Paulo: atualização 2011. Arq Bras Cardiol. 2012;99(2):755-61.

23. Baert V, Gorus E, Mets T, Bautmans I. Motivators and barriers for physical activity in older adults with osteoporosis. J Geriatr Phys Ther. 2015;38(3):105-14.

24. Haskell WL, Lee I-M, Pate RR, Powell KE, Blair SN, Franklin BA, et al. Physical activity and public health: updated recommendation for adults from the American College of Sports Medicine and the American Heart Association. Circulation. 2007;116(9):1081-93.

25. World Health Organization. Global recommendations on physical activity for health. Geneva: WHO; 2010.

26. Hallal PC, Reis RS, Hino AAF, Santos MS, Grande D, Krempel MC, et al. Avaliação de programas comunitários de promoção da atividade física: o caso de Curitiba, Paraná. Rev Bras Ativ Fís Saúde. 2009;14(2):104-14.

27. Fórum Brasileiro de Segurança Pública. Anuário Brasileiro de Segurança Pública. São Paulo; 2016.

28. Salvador EP, Florindo AA, Reis RS, Costa EF. Percepção do ambiente e prática de atividade física no lazer entre idosos. Rev Saúde Pública. 2009;43(6):972-80.

29. Inácio RF, Salvador EP, Florindo AA. Análise descritiva da prática de atividade física no lazer de idosos residentes em uma região de baixo nível socioeconômico da zona leste de São Paulo, SP. Rev Bras Ativ Fís Saúde. 2011;16(2):150-5.

30. Aguiar B, Moraes H, Silveira H, Oliveira N, Deslandes AC, Laks J. Efeito do treinamento físico na qualidade de vida em idosos com depressão maior. Rev Bras Ativ Fis Saúde. 2014;19(2):205-14.

31. King AC. Interventions to promote physical activity by older adults. J Gerontol A Biol Sci Med Sci. 2001;56(Suppl 2):3646.

32. Giraldo A, Gomes G, Serafim T, Zorzeto L, Aquino D, Kokubun E. Influência de um programa de exercícios físicos no uso de serviços de saúde na Atenção Básica de Saúde do município de Rio Claro, SP. Rev Bras Ativ Fis Saúde. 2013;18(2):18696.

\section{Endereço para correspondência:}

Wesley Lessa Pinheiro

Universidade Federal do Ceará

Faculdade de Medicina

Rua professor Costa Mendes, 1608, Bloco Didático, $5^{\circ}$ Andar

Bairro: Rodolfo Teófilo

CEP: 60430-140 - Fortaleza - CE - Brasil

E-mail: wesleylpinheiro@gmail.com 